\title{
Sociological Methods \& Research
}

http://smr.sagepub.com

\section{Conditions for the Equivalence of the Autoregressive Latent Trajectory Model and a Latent Growth Curve Model With Autoregressive Disturbances \\ Ellen L. Hamaker \\ Sociological Methods Research 2005; 33; 404 \\ DOI: $10.1177 / 0049124104270220$}

The online version of this article can be found at:

http://smr.sagepub.com/cgi/content/abstract/33/3/404

\section{Published by:}

(5)SAGE Publications

http://www.sagepublications.com

Additional services and information for Sociological Methods \& Research can be found at:

Email Alerts: http://smr.sagepub.com/cgi/alerts

Subscriptions: http://smr.sagepub.com/subscriptions

Reprints: http://www.sagepub.com/journalsReprints.nav

Permissions: http://www.sagepub.com/journalsPermissions.nav

Citations (this article cites 4 articles hosted on the

SAGE Journals Online and HighWire Press platforms):

http://smr.sagepub.com/cgi/content/refs/33/3/404 


\title{
Conditions for the Equivalence of the Autoregressive Latent Trajectory Model and a Latent Growth Curve Model With Autoregressive Disturbances
}

\author{
ELLEN L. HAMAKER \\ University of Amsterdam
}

\begin{abstract}
Curran and Bollen combined two models for longitudinal panel data: the latent growth curve model and the autoregressive model. In their model, the autoregressive relationships are modeled between the observed variables. This is a different model than a latent growth curve model with autoregressive relationships between the disturbances. However, when the autoregressive parameter $\rho$ is invariant over time and lies between -1 and 1 , it can be shown that these models are algebraically equivalent. This result can be shown to generalize to the multivariate case. When the autoregressive parameters in the autoregressive latent trajectory model vary over time, the equivalence between the autoregressive latent trajectory model and a latent growth curve model with autoregressive disturbances no longer holds. However, a latent growth curve model with time-varying autoregressive parameters for the disturbances could be considered an interesting alternative to the autoregressive latent trajectory model with time-varying autoregressive parameters.
\end{abstract}

Keywords: latent growth curve model; autoregressive latent trajectory model; autoregression; structural equation modeling

In structural equation modeling (SEM) literature, two important classes of models to analyze longitudinal panel data can be distinguished. The oldest consists of models containing autoregressive relations, such as the simplex model, the quasi-simplex model, the Wiener-simplex model, and the Markov model (Guttman 1954; Jöreskog 1971, 1979; Humphreys 1960; Anderson 1960). Characteristic of these autoregressive models is that the current observations are regressed on the prior observation of the same variable, and the

AUTHOR'S NOTE: I would like to thank Conor V. Dolan and Peter C. Molenaar for their helpful comments during the preparation of this manuscript. 
further apart (in time) two observations are, the smaller the connection between them. Put differently, the correlation structure is characterized by a decrease in correlations as they are further away from the main diagonal. Although not customary, these models can be extended with structured means (Browne and Du Toit 1991; Mandys, Dolan, and Molenaar 1994).

A more recently developed class of models to analyze longitudinal panel data concentrates on both the covariance structure and the means. It can be understood as the longitudinal version of the multilevel model and goes by names such as the latent growth curve (LGC) model, level and shape model, latent trajectory model, random effects model, and random coefficients modeling (Aitkin and Longford 1986; Duncan et al. 1999; Meredith and Tisak 1984, 1990). These models are used to describe growth curves while allowing individuals to differ from each other with respect to their individual growth curve parameters. What is estimated in these models are the means and (co)variances across subjects of these intraindividual curve parameters.

Curran and Bollen (2001) integrated the LGC model and the autoregressive model in what they called the autoregressive latent trajectory (ALT) model (for the technical details, see Bollen and Curran 2004). This model consists of a standard LGC model with autoregressive relationships between the observed variables. This model differs from LGC models with autocorrelated disturbances, such as proposed by Chi and Reinsel (1989). In this article, it is shown that, under certain conditions, the LGC model with autoregressive relationships between the observed variables and the LGC model with autoregressive relationships between the disturbances are algebraically equivalent. The necessary conditions concern the invariability of the autoregressive parameter $\rho$ over time, and limitations on the value that this parameter can take on (i.e., $|\rho|<1$ ). The relationship between the parameters in the two model formulations and their function is elaborated on. In addition, it is shown how the means, variances, and covariances of the intraindividual curve parameters in the two representations are related to each other. Also, it is shown that the model equivalence generalizes to the multivariate case. In the Discussion section, some additional remarks are made about more general models that include growth curves and autoregressive parameters. 


\section{EQUIVALENT REPRESENTATIONS OF THE SAME UNIVARIATE TIME SERIES OF A SINGLE SUBJECT}

In this section, the univariate ALT model of Bollen and Curran (2004; Curran and Bollen 2001) is presented for a single subject. It is shown that under certain conditions, this model can be rewritten as an LGC model with autoregressive disturbances. The function that the intraindividual curve parameters have in both model formulations is illustrated with a numerical example.

\section{ALT MODEL WITH TIME-INVARIANT $\rho$ AND $|\rho|<1$}

Consider the specific version of the ALT model in which the autoregressive parameter $\rho$ is invariant over time. Using $i$ as the subject index, the model for the observations is

$$
y_{t, i}=\alpha_{i}+t \beta_{i}+\rho y_{t-1, i}+\epsilon_{t, i},
$$

where $t$ is the occasion $(t=-\infty, \ldots,-1,0,1, \ldots, \infty), \alpha_{i}$ is a constant, $\beta_{i}$ is the regression coefficient by which the current $y$ is regressed on time, $\rho$ is the autoregressive parameter by which the current $y$ is regressed on the previous $y$, and $\epsilon_{t, i}$ is a residual. The series $\epsilon_{t}$ is a white noise sequence, meaning that the residuals $\epsilon$ are independently and identically distributed, with $\epsilon_{t} \sim \mathrm{N}\left[0, \sigma_{\epsilon}^{2}\right]$. As can be seen from equation (1), the LGC parameters $\beta$ and $\alpha$ contain a subject index, indicating that they differ across subjects, whereas the autoregressive parameter $\rho$ is invariant across subjects.

The model in equation (1) is a recursive expression in that the current $y$, which is a function of $\alpha_{i}$ and $\beta_{i}$, is also regressed on the previous $y$, which is in turn a function of $\alpha_{i}$ and $\beta_{i}$, as well as of the $y$ preceding it, and so on. Because of this recursion, the process has to be "started up" if one wishes to use this model in practice. In Bollen and Curran (2004:364), it is shown that under the restriction that $|\rho|<1$, the expression for $y_{0, i}$ is

$$
y_{0, i}=\alpha_{i}(1-\rho)^{-1}-\beta_{i} \rho(1-\rho)^{-2}+z_{0, i},
$$

where $z_{0, i}=\sum_{j=0}^{\infty} \rho^{j} \epsilon_{-j, i}$; that is, $z_{0, i}$ is an infinite weighted sum of previous residuals $\epsilon \mathrm{S}$. This $z_{0, i}$ is elaborated on below. After the 
process is started up at $t=0$, the model defined in equation (1) can be used for the following occasions (i.e., for $t=1,2, \ldots$ ). The nonlinear constraints $1 /(1-\rho)$ for $\alpha_{i}$ and $\rho /(1-\rho)^{2}$ for $\beta_{i}$ at $t=0$ may lead to computational problems when SEM software is used to fit this model. ${ }^{1}$ To avoid the use of these nonlinear constraints, one can treat the first observation $y_{0, i}$ as predetermined (Bollen and Curran 2004). However, as Bollen and Curran (2004) pointed out, this has the effect that the standard LGC model is no longer nested under the ALT model. To establish nesting of the LGC model under this predetermined version of the ALT model, they proposed to treat the first observation in the LGC model also as predetermined.

\section{ALTERNATIVE EXPRESSION}

Here it is shown that by rewriting the ALT model as an LGC model with autoregressive disturbances, the recursiveness is separated from the trend. The latter implies that the process is much easier to start up. To obtain this alternative expression, we recursively enter the expression of the previous observation ${ }^{2}$ into equation (1); that is,

$$
\begin{aligned}
y_{t, i}= & \alpha_{i}+t \beta_{i}+\rho y_{t-1, i}+\epsilon_{t, i} \\
= & \alpha_{i}+t \beta_{i}+\rho\left(\alpha_{i}+(t-1) \beta_{i}+\rho y_{t-2, i}+\epsilon_{t-1, i}\right)+\epsilon_{t, i} \\
= & \alpha_{i}+\rho \alpha_{i}+t \beta_{i}+(t-1) \rho \beta_{i}+\rho^{2} y_{t-2, i}+\rho \epsilon_{t-1, i}+\epsilon_{t, i} \\
= & \alpha_{i}+\rho \alpha_{i}+t \beta_{i}+(t-1) \rho \beta_{i} \\
& +\rho^{2}\left(\alpha_{i}+(t-2) \beta_{i}+\rho y_{t-3, i}+\epsilon_{t-2, i}\right)+\rho \epsilon_{t-1, i}+\epsilon_{t, i} \\
= & \alpha_{i}+\rho \alpha_{i}+\rho^{2} \alpha_{i}+t \beta_{i}+(t-1) \rho \beta_{i} \\
& +(t-2) \rho^{2} \beta_{i}+\rho^{3} y_{t-3, i}+\rho^{2} \epsilon_{t-2, i}+\rho \epsilon_{t-1, i}+\epsilon_{t, i} \\
\cdots & \\
= & \sum_{j=0}^{\infty} \rho^{j} \alpha_{i}+\sum_{j=0}^{\infty}(t-j) \rho^{j} \beta_{i}+\sum_{j=0}^{\infty} \rho^{j} \epsilon_{t-j, i} .
\end{aligned}
$$

The last term in equation (3) may be recognized as a moving average (MA) process of infinite order. It is a well-known fact that rewriting a first-order autoregressive (AR(1)) process as an MA process results in such an expression (e.g., Box and Jenkins 1976; Chatfield 1989; 
Hamilton 1994). We make use of this result and replace the last term in equation (3) by $z_{t, i}$, which is an $\mathrm{AR}(1)$ process; that is,

$$
z_{t, i}=\rho z_{t-1, i}+\epsilon_{t, i}=\sum_{j=0}^{\infty} \rho^{j} \epsilon_{t-j, i},
$$

with $z_{t} \sim \mathrm{N}\left(0, \sigma_{z}^{2}\right)$. Note that as a result of autoregression, these $z \mathrm{~s}$ are not independent over $t$, in contrast to the $\epsilon \mathrm{s}$. The expression in equation (3) can be further simplified by use of the geometric series in the following way:

$$
\begin{aligned}
y_{t, i} & =\sum_{j=0}^{\infty} \rho^{j} \alpha_{i}+\sum_{j=0}^{\infty}(t-j) \rho^{j} \beta_{i}+z_{t, i} \\
& =\alpha_{i} \sum_{j=0}^{\infty} \rho^{j}+\beta_{i} \sum_{j=0}^{\infty}(t-j) \rho^{j}+z_{t, i} \\
& =\alpha_{i} \sum_{j=0}^{\infty} \rho^{j}+\beta_{i}\left\{\sum_{j=0}^{\infty} t \rho^{j}-\sum_{j=0}^{\infty} j \rho^{j}\right\}+z_{t, i} \\
& =\alpha_{i} \sum_{j=0}^{\infty} \rho^{j}-\beta_{i} \sum_{j=0}^{\infty} j \rho^{j}+t \beta_{i} \sum_{j=0}^{\infty} \rho^{j}+z_{t, i} \\
& =\alpha_{i} \sum_{j=0}^{\infty} \rho^{j}-\beta_{i} \rho \sum_{j=0}^{\infty} j \rho^{j-1}+t \beta_{i} \sum_{j=0}^{\infty} \rho^{j}+z_{t, i} \\
& =\alpha_{i}(1-\rho)^{-1}-\beta_{i} \rho(1-\rho)^{-2}+t \beta_{i}(1-\rho)^{-1}+z_{t, i} \\
& =\delta_{i}+t \gamma_{i}+z_{t, i} .
\end{aligned}
$$

The model in equation (5) can be recognized as an LGC model, where $\delta_{i}$ is the intercept of individual $i$, which can be written as a function of $\alpha_{i}, \beta_{i}$, and $\rho$; that is,

$$
\delta_{i}=\alpha_{i}(1-\rho)^{-1}-\beta_{i} \rho(1-\rho)^{-2},
$$

and $\gamma_{i}$ is the slope parameter of subject $i$, which can be written as a function of $\beta_{i}$ and $\rho$; that is,

$$
\gamma_{i}=\beta_{i}(1-\rho)^{-1} .
$$

Thus, it has been shown that, if the autoregressive parameter $\rho$ does not vary over time and $|\rho|<1$, the ALT model can be rewritten 
as an LGC model (equation (5)) with autoregressive disturbances (equation (4)). Because the recursiveness is separated from the trend in this formulation, the process is easier to start up when applied in practice; that is,

$$
y_{0, i}=\delta_{i}+z_{0, i} \text {. }
$$

Note that this $z_{0, i}$ is identical to the one in equation (2), where it was equated to an infinite weighted sum of previous $\epsilon \mathrm{S}$. This relationship between $z_{0, i}$ and the previous $\epsilon \mathrm{S}$ can also be obtained from equation (4) with $t=0 .{ }^{3}$ From equation (8), it is clear that to use the model defined in equations (4) and (5) in practice, one does not need the nonlinear constraints as defined in equation (2), nor does one have to treat the first observation as predetermined.

\section{THE FUNCTION OF THE PARAMETERS $\alpha_{i}, \beta_{i}, \delta_{i}, \gamma_{i}$, AND $\rho$}

The algebraic relationships between the parameters $\alpha_{i}$ and $\beta_{i}$ in equation (1) and the parameters $\delta_{i}$ and $\gamma_{i}$ in equations (4) and (5) are given in equations (6) and (7). It can be seen that these relationships are relatively complicated. To gain more insight into the roles that these parameters play, a numerical example is given. The subject index $i$ is omitted as it plays no role here. In Table 1 , a series $\epsilon_{1}$ to $\epsilon_{8}$ was generated. In addition, a value for $z_{0}$ was randomly chosen. Then, $\rho$ was fixed at .8, $\alpha$ at 1 , and $\beta$ at .1. Through use of equations (6) and (7), it was determined that $\delta=3$ and $\gamma=.5$. Next, the values for $y_{0}$ to $y_{8}$ were calculated using equations (4) and (5) (see columns 3 and 4 in Table 1). To show that the model in equations (4) and (5) leads to the same results as the model in equations (1) and (2), $y_{0}$ was calculated using equation (2) (to start up the process), and from there on, the values for $y_{1}$ to $y_{8}$ were calculated using equation (1) (see column 5 in Table 1). It can be seen that these two methods lead to the same values for $y$.

The values of $y$ are plotted in Figure 1 along with the linear trend based on the intercept $\delta=3$ and slope $\gamma=.5$. It can be seen that the parameters $\delta$ and $\gamma$ describe the deterministic trend around which the observations are scattered. Regardless of the value of $\rho$, these parameters have the same role as the parameters in standard LGC models (i.e., $\delta$ is the individual's intercept, and $\gamma$ is the individual's 
TABLE 1: Numeric Example Showing the Equivalence Between the Two Model Formulations With $\rho=.8$

\begin{tabular}{ccccc}
\hline & & \multicolumn{2}{c}{$\begin{array}{c}\text { Equations (4) and (5) } \\
\delta=3 \text { and } \gamma=.5\end{array}$} & $\begin{array}{c}\text { Equations (1) and (2) } \\
\alpha=1 \text { and } \beta=.1\end{array}$ \\
\cline { 3 - 4 } \cline { 5 - 5 } $\mathrm{t}$ & $\epsilon$ & $\mathrm{z}$ & $\mathrm{y}$ & $\mathrm{y}$ \\
\hline 0 & & 1.1246088 & 4.124609 & 4.124609 \\
1 & -1.1465057 & -0.2468187 & 3.253181 & 3.253181 \\
2 & -1.5135502 & -1.7110052 & 2.288995 & 2.288995 \\
3 & 0.4281170 & -0.9406871 & 3.559313 & 3.559313 \\
4 & 0.4667663 & -0.2857833 & 4.714217 & 4.714217 \\
5 & 0.9058520 & 0.6772253 & 6.177225 & 6.177225 \\
6 & 0.7102752 & 1.2520555 & 7.252055 & 7.252055 \\
7 & -0.2106597 & 0.7909847 & 7.290985 & 7.290985 \\
8 & 2.4574620 & 3.0902498 & 10.090250 & \\
\hline
\end{tabular}

NOTE: The first column indicates time. The second column contains the i.i.d. disturbances $\epsilon$ that were generated. The first element of columns $3, z_{0}$, was also randomly chosen and used in both model formulations. All other elements in the third column (i.e., $z_{1}$ to $z_{8}$ ) were then obtained by use of equation (4). Using equation (5) gave the values for $y$ in column 4 . The fifth column contains $y$ based on equations (1) and (2).

slope). In contrast, the parameter values $\alpha=1$ and $\beta=.1$ do not have this simple function in the data plotted in Figure 1: Only when $\rho=0$ do $\alpha$ and $\beta$ serve as the intercept and slope (see also equations (6) and (7)).

Although the autoregressive parameter $\rho$ is identical in both model specifications, its function differs. In the ALT model, it serves the same role as in the original autoregressive models: In these models, $\rho$ is the parameter by which $y_{t}$ is regressed on $y_{t-1}$, representing the dependency of the current observation on the previous observation. In contrast, in the LGC model with $\mathrm{AR}(1)$ disturbances, $\rho$ is the parameter by which the current disturbance $z_{t}$ is regressed on the previous disturbance. This latter role of $\rho$ may be more difficult to comprehend: The AR(1) disturbances imply that, although the unconditional expectation of $z_{t}$ is zero (i.e., $\mathrm{E}\left[z_{t}\right]=0$ ), the expectation of $z_{t}$ conditional on $z_{t-1}$ is not zero but can be expressed as $\mathrm{E}\left[z_{t} \mid z_{t-1}\right]=\rho z_{t-1}$. From this conditional expectation, it can be seen that, when $\rho>0$ (as is the case in this example), this implies that if the observation at a certain occasion $t$ lies above the deterministic trend (i.e., $z_{t}>0$ ), the value of $y_{t+1}$ is also likely to lie above the deterministic trend, 


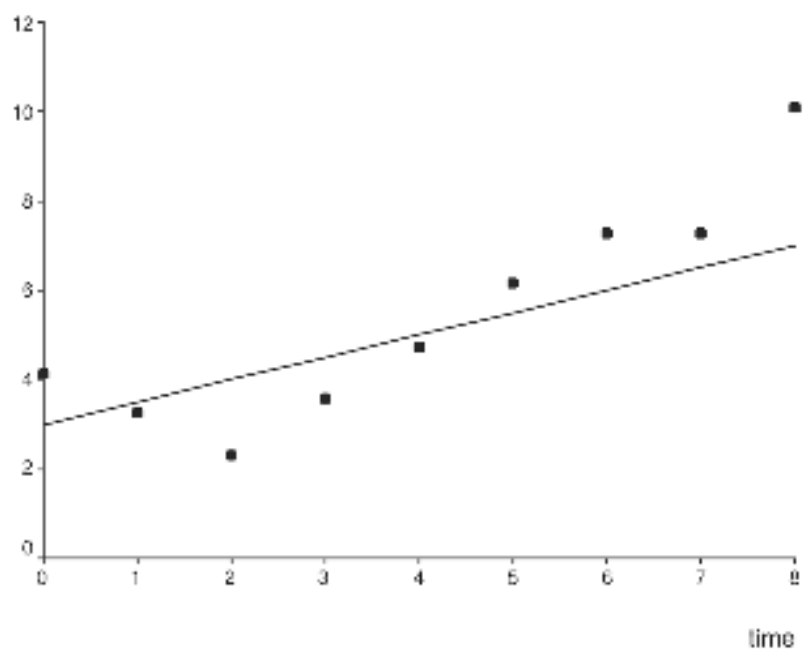

\section{Figure 1: Data From Table 1}

NOTE: Dots represent values of $y$ at different occasions, and the straight line represents deterministic trend with intercept $\delta=3$ and slope $\gamma=.5$.

as $\mathrm{E}\left[z_{t+1} \mid z_{t}\right]=\rho z_{t}>0$. Similarly, if $y_{t}$ lies below the deterministic trend, a $\rho>0$ results in the expectation that $y_{t+1}$ will also lie below the deterministic trend. This is nicely illustrated in Figure 1. If, in contrast, $\rho<0$, this implies that when $y_{t}$ lies above the deterministic trend (and so $z_{t}>0$ ), $y_{t+1}$ is likely to lie below it, as $\mathrm{E}\left[z_{t+1} \mid z_{t}\right]=\rho z_{t}<0$, and vice versa.

\section{MULTIPLE SUBJECTS}

In standard LGC modeling, individuals are allowed to differ from each other with respect to their trend parameters. Rather than to estimate each individual's trend parameters, one is interested in the means, variances, and covariances (across individuals) of these intraindividual trend parameters. In this section, it is shown how the means, variances, and covariance of the trend parameters $\gamma_{i}$ and $\delta_{i}$ can be written as functions of the means, variances, and covariance of the parameters $\beta_{i}$ and $\alpha_{i}$. 
The mean and variance of the intraindividual intercept $\delta_{i}$ can be expressed as functions of the means and variances of the intraindividual constant $\alpha_{i}$ and the intraindividual regression coefficient $\beta_{i}$ and of $\rho$; that is,

$$
\begin{aligned}
\mu_{\delta}= & \mathrm{E}\left[\delta_{i}\right]=\mu_{\alpha}(1-\rho)^{-1}-\rho \mu_{\beta}(1-\rho)^{-2}, \\
\sigma_{\delta}^{2}= & \mathrm{E}\left[\left\{\delta_{i}-\mu_{\delta}\right\}^{2}\right]=\sigma_{\alpha}^{2}(1-\rho)^{-2}+\rho^{2} \sigma_{\beta}^{2}(1-\rho)^{-4} \\
& -2 \rho \sigma_{\alpha \beta}(1-\rho)^{-3} .
\end{aligned}
$$

Similarly, the mean and variance of the slope $\gamma_{i}$ can now be written as a function of the mean and variance of the intraindividual regression parameter $\beta_{i}$ and the autoregressive parameter $\rho$ as follows:

$$
\begin{aligned}
\mu_{\gamma} & =\mathrm{E}\left[\gamma_{i}\right]=\mu_{\beta}(1-\rho)^{-1}, \\
\sigma_{\gamma}^{2} & =\mathrm{E}\left[\left\{\gamma_{i}-\mu_{\gamma}\right\}^{2}\right]=\sigma_{\beta}^{2}(1-\rho)^{-2} .
\end{aligned}
$$

Finally, the covariance between $\delta_{i}$ and $\gamma_{i}$ expressed as a function of the (co)variances of $\alpha_{i}, \beta_{i}$, and $\rho$ results in

$$
\sigma_{\gamma \delta}=\mathrm{E}\left[\left\{\gamma_{i}-\mu_{\gamma}\right\}\left\{\delta_{i}-\mu_{\delta}\right\}\right]=\sigma_{\alpha \beta}(1-\rho)^{-2}-\rho \sigma_{\beta}^{2}(1-\rho)^{-3} .
$$

From equation (10), it can be seen that, even when there is no variation in intraindividual constant $\alpha_{i}$ (i.e., $\sigma_{\alpha}^{2}=0$ and $\sigma_{\alpha \beta}=0$ ), this does not imply that the individuals have the same intercept $\delta_{i}$, unless the autoregressive parameter is zero $(\rho=0)$ and/or all individuals have the same regression coefficient $\beta$ (i.e., $\sigma_{\beta}^{2}=0$ ). Similarly, from equation (13), it can be seen that, even when the constant $\alpha_{i}$ and the regression coefficient $\beta_{i}$ are not correlated (i.e., $\sigma_{\alpha \beta}=0$ ), the intercept $\delta_{i}$ and the slope $\gamma_{i}$ may still be correlated (as long as $\rho$ and $\sigma_{\beta}^{2}$ are not zero).

\section{MULTIVARIATE GENERALIZATION}

Bollen and Curran also considered multivariate ALT models (Bollen and Curran 2004; Curran and Bollen 2001). Using $i$ as subject index, a $k$-variate ALT model with time-invariant auto- and cross-regressive parameters can be written as

$$
\mathbf{Y}_{t, i}=\mathbf{A}_{i}+\mathbf{B}_{i} t+\mathbf{R} \mathbf{Y}_{t-1, i}+\mathbf{E}_{t, i},
$$


where $\mathbf{Y}_{t, i}$ is a $k \times 1$ vector with the observations of subject $i$ at occasion $t, \mathbf{A}_{i}$ is a $k \times 1$ vector with constants, $\mathbf{B}_{i}$ is a $k \times 1$ vector with regression coefficients to regress the current observations on time, $t$ is a scalar indicating the occasion, $\mathbf{R}$ is a $k \times k$ matrix with auto- and cross-regression coefficients, and $\mathbf{E}_{t, i}$ is a $k \times 1$ vector with residuals.

Analogue to the univariate case, this multivariate ALT model can be rewritten as a multivariate LGC model with auto- and crossregressive disturbances, under the restriction that the eigenvalues of $\mathbf{R}$ lie between -1 and 1 . Some algebra shows ${ }^{4}$ that equation (14) is equivalent to

$$
\mathbf{Y}_{t, i}=\Delta_{i}+\Gamma_{i} t+\mathbf{Z}_{t, i}
$$

where $\Delta_{i}$ is a $k \times 1$ vector with intercepts, which are a function of $\mathbf{A}_{i}, \mathbf{B}_{i}$, and $\mathbf{R}$; that is,

$$
\boldsymbol{\Delta}_{i}=(\mathbf{I}-\mathbf{R})^{-1} \mathbf{A}_{i}-(\mathbf{I}-\mathbf{R})^{-2} \mathbf{R} \mathbf{B}_{i},
$$

where $\Gamma_{i}$ is a $k \times 1$ vector with slopes, which can be written as a function of $\mathbf{B}_{i}$ and $\mathbf{R}$; that is,

$$
\Gamma_{i}=(\mathbf{I}-\mathbf{R})^{-1} \mathbf{B}_{i} .
$$

and $\mathbf{Z}_{t, i}$ is a first-order vector autoregressive model, which is identical to a vector moving average model of infinite order (Hamilton 1994:260); that is,

$$
\mathbf{Z}_{t, i}=\mathbf{R} \mathbf{Z}_{t-1, i}+\mathbf{E}_{t, i}=\sum_{j=0}^{\infty} \mathbf{R}^{j} \mathbf{E}_{t-j, i}
$$

As the recursion (equation (18)) is separated from the trend (equation (15)), this model formulation does not require specific constraints to start up the process when used in practice.

\section{DISCUSSION}

In this article, it is shown that, given time-invariant autoregressive parameters that satisfy $|\rho|<0$, the ALT model formulated by Bollen and Curran (2004; Curran and Bollen 2001) is equivalent to an LGC model with AR(1) disturbances. Formulating the model as an LGC 
with $\mathrm{AR}(1)$ disturbances results in a separation of the trend from the recursiveness in the model. This has two advantages. First, the parameters $\delta_{i}$ and $\gamma_{i}$ have the same function as the trend parameters in standard LGC modeling: $\delta_{i}$ is the individual's intercept, and $\gamma_{i}$ is the individual's slope, which describes the deterministic trend of subject $i$. Second, the process does not need to be started up with nonlinear constraints that may lead to computational problems.

In this article, only univariate ALT models with time-invariant autoregressive parameter $\rho$ and $|\rho|<1$ were considered. Bollen and Curran (2004) proposed a more general version of the ALT model, in which the autoregressive parameters can vary over time and may exceed unity in absolute value. This model can be obtained from equation (1) by replacing the time-invariant $\rho$ by $\rho_{t, t-1}$, relating $y_{t, i}$ to $y_{t-1, i}$. This more general model is not equivalent to an LGC model with $\mathrm{AR}(1)$ disturbances. The reason for this is that if the $\rho$ s vary over time and/or exceed unity in absolute value, one cannot use the geometric series and the expression of an AR(1) process as an MA of infinite order. Both results are essential in establishing the equivalence between these models.

However, one can also consider an LGC model with autocorrelated disturbances in which the autoregressive parameters vary over time. Such a model can be obtained from the model in equations (4) and (5) by replacing the time-invariant $\rho$ in equation (4) by a timespecific $\rho_{t, t-1}$, which relates the distance of the observation $y_{t, i}$ to the deterministic trend at occasion $t$ (i.e., $z_{t, i}$ ) to the distance of the observation $y_{t-1, i}$ to the trend at the previous occasion (i.e., $z_{t-1, i}$ ). As with the LGC models discussed in this article, this extension with time-varying autoregressive parameters does not require to be started up. In contrast, as Bollen and Curran (2004) pointed out, the ALT model with time-varying $\rho$ s always requires the first observation to be treated as predetermined. To this end, three extra parameters must be estimated (i.e., the covariances between $y_{0, i}$ and the parameters $\alpha_{i}$ and $\beta_{i}$, as well as the mean of $\left.y_{0, i}\right)$. Hence, although the ALT model with time-varying autoregressive parameters and an LGC model with autocorrelated disturbances and varying autoregressive parameters are not equivalent, the latter could be considered as an interesting and more parsimonious alternative to the former in cases in which the model with time-invariant $\rho$ s does not fit. 


\section{NOTES}

1. Such computational problems were encountered in LISREL (Jöreskog and Sörbom 1999) but not in Mx (Neale et al. 1999). The Mx-input files, based on an empirical example for both model formulation discussed in this article, can be obtained from http://users.fmg.uva.nl/ehamaker/.

2. Some readers may feel uncomfortable with the resulting expressions going back in time to minus infinity. However, the alternative expression arrived at in equation (3) is mathematically equivalent to the expression given in equation (1). Either expression is only used as a local description of some process. Hence, it is not implied that the actual process follows this description to infinity or has been following it from minus infinity.

3. The variance across subjects of this $z_{0, i}$ is not the same as the variance of the disturbances $\epsilon_{t, i}$, but this relationship is $\sigma_{z}^{2}=\sigma_{\epsilon}^{2} /\left(1-\rho^{2}\right)$. Although not necessary, the latter may be used as an constraint in model fitting. This constraint could be used in model specification based on equations (1) and (2) as well as model specification based on equations (4) and (8).

4. For details, see http://users.fmg.uva.nl/ehamaker/.

\section{REFERENCES}

Aitkin, Murray, and Nicholas T. Longford. 1986. "Statistical Modelling Issues in School Effectiveness Studies." Journal of the Royal Statistical Society, Series A (General) 149:1-43.

Anderson, Theodore W. 1960. "Some Stochastic Process Models for Intelligence Test Scores." Pp. 205-20 in Mathematical Methods in the Social Sciences, 1959, edited by K. J. Arrow, S. Karlin, and P. Suppes. Stanford, CA: Stanford University Press.

Bollen, Kenneth A., and Patrick J. Curran. 2004. "Autoregressive Latent Trajectories (ALT) Models: A Synthesis of Two Traditions." Sociological Methods \& Research 32:336-83.

Box, George E. P., and Gwilym M. Jenkins. 1976. Time Series Analysis: Forecasting and Control. Rev. ed. San Francisco: Holden-Day.

Browne, Michael W., and Stephen H. C. Du Toit. 1991. "Models for Learning Data." Pp. 47-68 in Best Methods for the Analysis of Change: Recent Advances, Unanswered Questions, Future Directions, edited by L. M. Collins and J. L. Horn. Washington, DC: American Psychological Association.

Chatfield, Christopher. 1989. The Analysis of Time Series: An Introduction. 4th ed. London: Chapman \& Hall.

Chi, Eric M., and Gregory C. Reinsel. 1989. "Models for Longitudinal Data With Random Effects and AR (1) Errors." Journal of the American Statistical Association 84:452-9.

Curran, Patrick J., and Kenneth A. Bollen. 2001. "The Best of Both Worlds: Combining Autoregressive and Latent Curve Models." Pp. 105-36 in New Methods for the Analysis of Change, edited by L. M. Collins and A. G. Sayer. Washington, DC: American Psychological Association.

Duncan, Terry E., Susan C. Duncan, Lisa A. Strycker, Fuzhong Li, and Anthony Alpert. 1999. An Introduction to Latent Variable Growth Curve Modeling: Concepts, Issues and Applications. Mahwah, NJ: Lawrence Erlbaum.

Guttman, L. A. 1954. "A New Approach to Factor Analysis: The Radex.” Pp. 197-218 in Mathematical Thinking in the Social Sciences, edited by P. F. Lazarsfeld. New York: Columbia University Press.

Hamilton, James D. 1994. Time Series Analysis. Princeton, NJ: Princeton University Press. 
Humphreys, Lloyd G. 1960. "Investigations of the Simplex." Psychometrika 25:313-23.

Jöreskog, Karl G. 1971. "Estimation and Testing of Simplex Models.” British Journal of Mathematical and Statistical Psychology 23:121-45.

1979. "Statistical Estimation of Structural Models in Longitudinal-Developmental Investigations.” Pp. 303-51 in Longitudinal Research in the Study of Behavior and Development, edited by J. R. Nesselroade and P. B. Baltus. New York: Academic Press.

Mandys, Frantisek, Conor V. Dolan, and Peter C. M. Molenaar. 1994. "Two Aspects of the Simplex Model: Goodness of Fit to Linear Growth Curve Structures and the Analysis of Mean Trends." Journal of Educational and Behavioral Statistics 19:201-15.

Meredith, William, and John Tisak. 1984. “'Tuckerizing' Curves.” Paper presented at the annual meeting of the Psychometric Society, Santa Barbara, CA. 1990. "Latent Curve Analysis." Psychometrika 55:107-22.

Neale, Michael C., Steven M. Boker, Gary Xie, and Hermine H. Maes. 1999. Mx: Statistical Modeling. 5th ed. Richmond, VA: Department of Psychiatry, Virginia Commonwealth University.

Ellen L. Hamaker works as a Ph.D. student in psychological methodology at the University of Amsterdam. Her research interests are time-series analysis and structural equation modeling. Previous publications on using structural equation modeling software for time-series analysis can be found in Structural Equation Modeling. 